\title{
Cómo Ella se convierte en "Loca". \\ Cuerpo y género en los significados sociales y morales de la enfermedad*
}

\author{
How She becomes "crazy". \\ Body and gender in the social and moral meanings of the disease \\ Como Ela se torna "louca". \\ Corpo e gênero nos significados sociais e morais da doença
}

\begin{abstract}
Anahi Sy**
Universidad Nacional de Lanús, CONICET, Buenos Aires, Argentina.

Correo electrónico: anahisy@gmail.com

Revista Corpo-grafías: Estudios críticos de y desde los cuerpos / Volumen 5 - Número 5 / Enero - diciembre de 2018 / ISSN impreso 2390-0288, ISSN digital 2590-9398 / Bogotá, D.C., Colombia / pp. 209-220.

Fecha de recepción: 19 de julio de 2017

Fecha de aceptación: 17 de agosto de 2017

Doi: https://doi.org/10.14483/25909398.14217

Cómo citar este artículo: Sy, Anahi. (2018, enero-diciembre). Cómo Ella se convierte en “Loca”. Cuerpo y género en los significados sociales y morales de la enfermedad. Revista Corpo-grafías: Estudios críticos de y desde los cuerpos, 5(5), p-p 209-220 / ISSN 2390-0288.

*Artículo de investigación: derivado de un acervo documental de 4058 historias clínicas provenientes del Hospital Psiquiátrico de mujeres "José Esteves" de la Provincia de Buenos Aires, Argentina, correspondientes al período 18951987.

**Doctora en Ciencias Naturales y Lic. En Antropología de la Universidad Nacional de la Plata. Investigadora y docente del Instituto de Salud Colectiva y la Universidad Nacional de Lanús. Investigadora CONICET, Buenos Aires, Argentina.
\end{abstract}

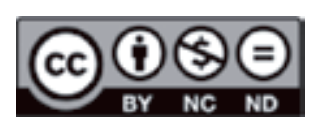




\section{Resumen}

Nuestro trabajo parte de un acervo documental de 4058 historias clínicas provenientes del Hospital Psiquiátrico de mujeres "José Esteves" de la Provincia de Buenos Aires (Argentina), correspondientes al período 1895-1987. Focalizamos en la historia clínica de una única mujer, cuya internación abarca casi el total de su vida adulta hasta su fallecimiento. El análisis, desde las categorías de género y "cuerpo individual, social y político" permite ilustrar la historia de muchas otras, funcionando como caso ejemplo sobre el modo en que la psiquiatría interviene en la configuración de las relaciones sociales y de género a lo largo del tiempo, delimitando signos y síntomas como atributos de enfermedad psíquica que conducen a invisibilizar la forma en que éstos expresan aquello que se considera "peligroso", "dañino", "anormal" para una mujer en determinado momento de la historia. Nuestro enfoque habilita la reflexión sobre los estereotipos de género que produce y reproduce la práctica médica hoy.

\section{Palabras clave: salud mental; cuerpo; género; historia clínica.}

\section{Abstract}

Our research analyzes continuities and changes that characterize the asylum model, between the years 1895-1987 from the analysis of a corpus of 4058 medical records belonging to Esteves Hospital of the Province of Buenos Aires, in Argentina. In this paper we focus on one unique case whose hospitalization goes almost her entire adult life till death. The analysis from Genre and "individual, social and political body" categories, able to visualize how psychiatry express changing and arbitrary nature of genre and social relations identified as diagnostic signs, at different times in history. Thus the analysis of the past, inquiring about how medical practice becomes a science of "real things" in appearance, enables discussion about present and future in mental health care.

Keywords: mental health; body; genre; medical record.

\section{Resumo}

Nosso trabalho é baseado em uma coleção documental de 4058 prontuários médicos do Hospital Psiquiátrico "José Esteves" da Província de Buenos Aires (Argentina), correspondente ao período de 1895-1987. Nós nos concentramos na história clínica de uma mulher solteira, cuja hospitalização cobre quase o total de sua vida adulta até a sua morte. A análise, a partir das categorias de gênero e "corpo individual, social e político", permite ilustrar a história de muitos outros, servindo de exemplo para o modo como a psiquiatria intervém na configuração das relações sociais e de gênero ao longo do tempo, definindo sinais e sintomas como atributos da doença psíquica que levam a invisibilizar a maneira como eles expressam o que é considerado "perigoso", "nocivo", "anormal" para uma mulher em determinado momento da história.

Nossa abordagem permite refletir sobre os estereótipos de gênero produzidos e reproduzidos pela prática médica atual.

Palavras-chave: saúde mental; corpo; gênero; história clínica. 


\section{Introducción}

La forma en que la psiquiatría en la actualidad construye y sostiene ciertos diagnósticos y tratamientos, siguiendo como guía fundamental el DSM5, tiende a negar la historicidad de la disciplina y, con ello, las continuidades y cambios que persisten en los signos, síntomas y terapias; generando la idea de estar tratando con «cosas reales», produciendo una cosificación biológica y física del sufrimiento mental.

Las historias clínicas en las que se basa este trabajo pertenecen al "Asilo de Alienadas de Lomas de Zamora" (creado en 1908), que a partir de 1976 adquiere su designación actual: Hospital Interzonal "José A. Esteves" (situado en Temperley, Provincia de Buenos Aires, Argentina). Se trata de mujeres que han fallecido en el hospital durante su internación, entre 1895 y 1987 . Dicha internación abarca en muchos casos, toda su vida adulta (40 o más años) lo cual implica grandes periodos de tiempo. Ello hace suponer la posibilidad de identificar cambios en la atención, conforme cambian las escuelas de pensamiento y modalidades de atención al sufrimiento mental, sin embargo, aparecen diversas continuidades -como la constante atribución de ciertos estereotipos de lo femenino a signos y síntomas psiquiátricos-, algunos cambios, con la aparición de psicofármacos que dan lugar al llamado "chaleco químico", como medio de contención que convive con "viejas" prácticas como el uso del chaleco de fuerza u otros medios de contención mecánica (Sy et al, 2016).

Focalizamos en la historia clínica de una única mujer, cuya internación abarca casi el total de su vida adulta hasta la muerte. El análisis permite ilustrar la historia de muchas otras, funcionando como caso ejemplo sobre el modo en que la psiquiatría interviene en la configuración de las relaciones sociales y de género a lo largo del tiempo, delimitando signos y síntomas como atributos de enfermedad psíquica.

En este sentido es pertinente reflexionar desde la categoría conceptual de género, en tanto construcción histórico social, que se reproduce a través del tiempo de distintas maneras y construye significados y subordinaciones que prevalecen en la religión, en los criterios médicos, científicos y en los dispositivos jurídicos; permitiendo visibilizar el carácter social y no biológico de los atributos de la masculinidad y la feminidad que toda sociedad elabora a partir de las diferencias de sexo, habilitando la reflexión sobre la forma en que tales diferencias se han constituido históricamente en desigualdad, en contextos socioculturales donde rigen valores patriarcales (Scott, 1990). Al respecto, no debe perderse de vista que la categoría género no debe invisibilizar en el análisis otras dimensiones como, raza, religión, clase social. Así, la categoría de género aparece entrecruzada con otros aspectos que intervienen en la construcción de la subjetividad humana (Burin y Meler, 2010), en este caso particular, los asilos de alienadas en general y el Asilo de Lomas en particular, si bien representaban espacios reservados a la locura de las mujeres, se confinó mayormente a mujeres, migrantes, pobres (si analizamos su composición poblacional, origen y ocupación) entonces, no es sólo el género sino también la clase social y el lugar de origen lo que atraviesa a la internación de estas mujeres, entre otras dimensiones posibles.

\section{Metodología}

Nuestro trabajo parte del análisis de un acervo documental de 4058 historias clínicas provenientes del Hospital Psiquiátrico de mujeres “José A. Esteves" de la Provincia de Buenos Aires (Argentina), correspondiente al período 1895-1987. Las Historias Clínicas a partir de las cuales elaboramos este trabajo pertenecen a mujeres que, inicialmente fueron internadas en el Hospital Nacional de Alienadas y, luego pasan al Asilo de Lomas (actual "Hospital Interzonal José Esteves") donde fallecen. 
El promedio de años total de estada en el hospital es de 19,4 y por años oscila entre los 12,6 para 1948 a 37,2 en 1982. En base a estos datos realizamos la lectura y análisis en profundidad de un número reducido de casos orientado a indagar sobre la vida de las mujeres durante su internación: cómo es admitida, cuáles son los registros de su voz y la de otro/s que intervienen en el ingreso; cuáles son las interacciones con el personal de salud durante su internación y los vínculos con el afuera (ya sea con la familia o allegados como con el sistema jurídico-legal que solicita la admisión), cuáles son los cuidados, tratamientos y evoluciones que registra el médico, enfermera o cualquier otro/s trabajador/es, qué lugar ocupa la voz de las mujeres en los registros, cuál es la escucha e interpretación de dicha voz. Con base en ello, en las páginas que siguen hemos elegido a "Ella" ${ }^{1}$ como la protagonista de una Historia, cuyo ingreso y recorrido en el hospital puede ser el de muchas o cualquiera de las mujeres internadas. El relato de Ella invita a conocer su ingreso desde las múltiples voces que intervienen: médicos, familia, juez y su propia voz y relaciones que establece hacia dentro y fuera de la institución, sus anhelos, frustraciones y tristezas, en suma la "lectura" e interpretación médica de todo ello, lo que justifica su internación hasta la muerte .

Es necesario aclarar que, si bien el relato que presentamos no proviene en su totalidad de la experiencia de una única mujer internada, tampoco se trata de ficción ${ }^{2}$. La composición del recorrido, las escenas y eventos que presentamos provienen de los resultados de la lectura analítica de diferentes experiencias y vivencias de las mujeres internadas registradas en las Historias Clínicas. Con esto no dejamos de considerar que si bien la experiencia subjetiva y de internación de cada una de estas mujeres es única y constituye un relato original, cada una también refleja algo de la realidad (Lewis, 1985), la época, la historia y lo social así como la multiplicidad de factores que atraviesan a la internación.

Presentar un recorrido de internación permite visualizar los diferentes factores y variables que se combinan e influyen de manera constante y simultánea desde la admisión hasta el fallecimiento como destino final del confinamiento de todas las mujeres internadas.

Sin embargo, reconocemos que éste como tantos otros escenarios permanecerán opacos en la medida en que no se logre trascender la historia individual, esto es, operar el pasaje de la escala "micro" a la escala "macro" de análisis. En este sentido, tomamos las categorías de género -tal como fuere desarrollada previamente- y la de los "tres cuerpos" como herramientas heurísticas de análisis para comprender e interpretar la internación de estas mujeres hasta la muerte. La propuesta desde la antropología por M. Lock y N. Schepper Hughes (1990) remite al cuerpo individual como aquel de la experiencia vivida, la relación con su entorno y sus afectos; el cuerpo social como símbolo de una "totalidad orgánica" cuando está sano o de una "desarmonía social" ante la enfermedad -recíprocamente la sociedad "sana" o "enferma" ofrece un modelo para estudiar el cuerpo- y, finalmente el cuerpo político se presenta como símbolo de disciplina y control, de gobierno individual y colectivo de los cuerpos en la reproducción, sexualidad, trabajo, aprendizaje y enfermedad, entre otras.

\section{Resultados}

\section{Ella}

A continuación presentamos la multiplicidad de "voces" y "miradas" que recaen sobre Ella, una mujer migrante española, que el 2 de agosto de 1924, con 39 años de edad, llega al hospital por orden judicial y, tras 44 años de internación, fallece el 11 de diciembre de 1968 , a los 83 años de edad.

Al abrir su historia clínica puede verse y olerse años de encierro, el primer documento que encontramos es un telegrama enviado al esposo de Ella:

1 Usamos el seudónimo "Ella" para garantizar el anonimato de las mujeres y de sus parientes, que serán nombrados descriptivamente por el grado de parentesco, ejemplo: Hija de Ella, Esposo de Ella, etc.

2 Oscar Lewis, precursor en los estudios sobre la pobreza en México, propone el término "realismo etnográfico" para distinguir su presentación de la cotidianeidad de familias con las que trabajó, del "realismo literario". En el primero los "días no están compuestos; son días reales y los individuos no son tipos imaginados, sino gente verdadera" (Lewis, 1985: 19). 


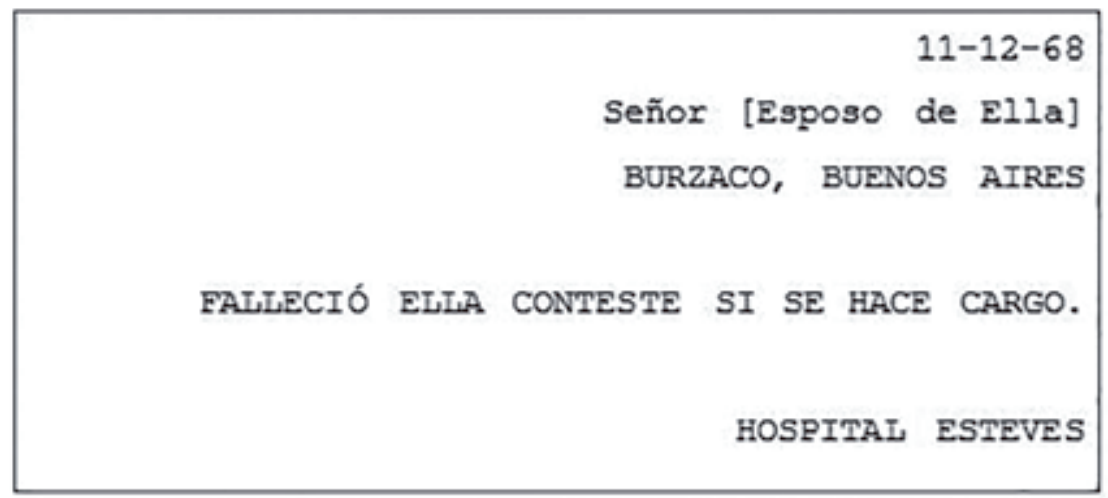

A continuación podemos ver con vida a Ella en la nota que narra su ingreso en el hospital:

El dia 25 del corriente, siendo las 7.45 se
presentó en la comisaria $17 \mathrm{ma}$ el agente Rodriguez
conduciendo detenida a la mujer Ella por cuanto en
la esquina de Libertad y Paraguay, habia oído que
aquella pronunciaba frases incoherentes como:
"actitudes de lucha por la vida", "gremialismo",
"sindicalismo", haciéndole presumir que tuviera
las facultades mentales alteradas.

La primera página de la historia clínica se encuentra atravesada por un sello de tinta roja con la inscripción: "INTERDICTA", lo que marca su internación por orden judicial.

Aparece en esta primera página un formulario que solicita consignar: 
Nombre y apellido Eua.

Nacionalidad Francesa

Residenciazurzaco

Profesión Empleadia.
Año de inqreso 1924

\section{Fecha de nacimiento [queda vacia para Ella]}

Estado Civil casada

Color: 3lanse

\section{Procedencia: [queda vacia para Ella] Localidad: 3urzaco}

Hij... de [queda vacia para Ella]

[Foto de ella]

Inqreso

Inqresa el 3 de ewero die 1924

Colocada por policisa.

\section{A disposición de [queda vacia para Ella]}

Diagnóstico ideas delinaness, alucinaciones avaitivas, Delinis sistematizado die persecucisn, Awcinacisw interpretativa.
[Ausencia de Foto]

Eqreso

\section{Altura del cuerpo 1,56}

peso 55 kilogramos

En la segunda página de la Historia Clínica puede observarse que lo que aparece completado por el médico que realiza la admisión es que la paciente no tiene antecedentes de locura-según dice su esposo-. No se ocupa de sus quehaceres domésticos", "desafectivizada con su marido y hasta con sus hijos".

Ella cuenta que fue despedida por sus patrones y desde entonces "por las cosas que se tejen en la sombra" no ha podido encontrar ocupación. "Relaciona su estado con la angustía que le produce el no hacer lo que ella quería (estudiar, ser útíl)". Por la calle la gente la mira "en forma significativa". Oye que le dicen "en voz alta, María la loca, María la loca" sus "mismos están confabulados con sus enemigos" y han pretendido hacerla pasar por loca. Internándola en el Hospital Nacional de Alienadas, le han dado muerte a su propia hijita, "etc., etc."
} 
Ingresa con una arden de la policia de la capital par lesiones en la via pública "Desprecian a su hija, la insultaban y le decian palabras ofensivas" A continuación aparece la siguiente descripción médica:

Mujer pequeña estatura. Buen esqueleto. Regular panículo adiposo. Piel blanca tinte rosada. Músculos bien desarrollados. Motilidad activa y fuerzas conservadas. Reflejos plantares en flexión. Los demás presentes e iguales a ambos lados. Audición y visión conservadas, pupilas iguales reaccionan a la luz y la acomodación MOP [Movimientos oculares de persecución] normal. Encías rosadas y dientes muy bien conservadas. Lengua húmeda y saburral Icapa fina de color blanquecino formada por bacterias que puede recubrir la superficie de la lengua. En la mayoría de los casos, la lengua saburral no tiene importancia y no provoca problemas de salud]. Tiroídes tamaño normal. Pulmones sonoridad normal. Respiración vesicular no se oye. Corazón tonos muy fuertes en todos los focos. Soplo sistólico en el foco pulmonar de muy escasa propagación, rítmo normal, pulso regular igual mediano frecuencia 140. Tensión MX180 Mn 100 (La enferma esta intranquila y con aspecto de terror). Abdomen: plano, paredes blandas, no se palpa nada anormal ni se despierta dolor. Orina D 1013 nada anormal. Urea en suero: 0,43\%0 Glucemia: 0,74\%0 Reacción Wassermann y Kahn: eritrosedimentación: 5

Diagnóstico: nerviosidad Eretismo cardio vascular [Med. Actividad muy intensa, y limitada en el tiempo, de un organismo a parte de él taquicardia inusual. Aumento de la tensión diferencial.

colesterol: $2,10 \% 0(\ldots)$

Continúa la descripción de aspectos somáticos o biomédicos sin advertir "anomalía" o malfuncionamiento orgánico o fisiológico alguno. A continuación aparece adjunta la siguiente nota:

Buenos aires, noviembre de 1925 Me dirijo al...... solicitándole se sirva autorizar al pie de la presente a esta dirección, si es $\circ$ no de su agrado, disponga se proceda a cortarle el cabello, en forma de uso actual, (melena) a su enferma........ puesto que ello resultaria para mayor facilidad de mantenimiento en condiciones higiénicas.

Saluda atentamente

El medico director

Doctor Julio G. Nogues 


\section{Señor medico Director \\ El que suscribe en carácter de 꼬 de la enferma de referencia, (comunica, autoriza) a la Dirección del Hospital Nacional de Alienadas que.... Se proceda a cortarle el cabello en la forma indicada en la solicitud que precede.}

\section{Firma: Tra de Ella}

Luego comienza el registro que da cuenta de la situación de la paciente durante la internación:

Enferma tranquila - durmió cuatro horas - se levantó bien - buena orientación en tiempo y lugar - memoría conservada -. Dice estar afónica desde hace meses, niega tener alucinaciones auditivas.

A casi un año de su ingreso en el hospital vuelve a registrarse algo sobre Ella: "a eso de las 12 se puso en extremo agresiva golpeando brutalmente a la enferma María A, siendo necesaria la aplicación del restraint"

A los pocos días

"intranquila, irritable.-reticente-negativista. Ideas persecutorias contra la familia "no me quieren porque estoy tisica" a veces observándose soliloquios-atenta al interrogatorio, deslizándose contadas veces ciertas incoherencias fugaces-algo erótica. Requiere repetidamente los medios contensivos. Sin conciencía de su estado."... pesa $50 \mathrm{~kg}$

A partir de ahí encontramos que los registros en la historia clínica ocurren alrededor del año, a propósito algún infortunio, si bien aparece como constante referencias previas a la situación de "La enferma" como es nombrada Ella:

"enferma muy excitable-agresiva-agresiva-incoherente- desorientada en tiempo y lugar- (...) la enferma pesa $49 \mathrm{lgg"}$

"memoria muy debilitada indiferente, muy incoherente risas inmotivadas desafectiva pesa $51 \mathrm{~kg} . "$

iud. cómo se llama? "Que se le importa" risas inmotivadas, un sentido de abstracción completa, no responde casi nada..."

El 30 de Abril de 1932 "presenta actítud indiferente toda pregunta que se le haga contesta: no sé-muy agresiva con las demas enfermas y con el personal del pabellón-caprichosa, desobediente-no trabaja en nada-sucia-come bien-duerme poco-soliloquios en voz baja -"...

Se repiten algunas notas que van a aparecer año a año: "nerviosa" "desafectíva" "respuesta muy pobre" "no colabora", "desaseada", "no trabaja". 
Aparece como novedad una consideración sobre su peso "a veces es necesario recurrír a la alímentación forzada pesa 44 y $1 / 2 \mathrm{Kg}$ ".

En 1933 "... Levantada se excita con suma frecuencia tornándose agresiva, revoltosa, coprolalica, obscena y obligando al uso del restraint" (...) "presta escasa atención, desorientada, respuestas incoherentes - memoría con fallas -... es visitada de vez en cuando desafectiva - déficit de sus facultades psiquicas - duerme con hipnóticos - se alimenta poco y merced a los reiterados esfuerzos del personal del pabellón"

A lo anterior se suma entre 1934 y 1960: “... exhibicionista (...) suele desnudarse o colocarse muchas prendas innecesarias, intranquila, agresiva, desaseada, no trabaja sucía destructoras indiferente, permanece vigílada en los patios por su tendencia a deambular. No atiende su aseo. Tendencia a la destrucción... poco visitada.

En 1960 "(...) salto la ventana del baño, se produce una herida superficial en codo derecho. Desinfección apósitos" (...) "Fisico discreto" (...) "examen endocrino".

Aparece una nueva categoría diagnóstica que parece condensar todos los antecedentes previos: "síndrome esquizofrénico".

En 1961 "... enferma desnutrida, anoréxica, pérdida de peso últimamente... se indica control de peso semanal y radiografía de tórax, anal de sangre."

En 1963 la enferma presenta antrax en pie derecho, se le prescribe penicilina

En 1964 se registra que "al no haber penicilina se sustítuye la medicación por criseosil 1 frasco ampolla cada 6 horas"

En 1965 "se le cae un banco sobre pie ocasionándole herida contuso cortantes...

En 1966 se registra la administración de Halopidol 3 C por día

\section{Continúa con golpes y recaidas entre 1970 y 1980}

En 1967 se registra: paciente desprolija. No colabora con el interrogatorío. Globalmente desorientada. Descenso de sus facultades intelectuales.

1968 Estacionaria. Desorientada negativista niega alucinaciones e ideas delirantes "con posterioridad a la muerte de mi híja yo observe y comprendi muchas cosas que antes había aceptado como hechos regidos por ellos"... "ya no tengo alucinaciones ni ilusiones" "he observado los hechos-los hechos" "querian matarme". -

11/12/1968 se constata el deceso de la paciente por paro cardio respiratorio no traumático.

No se presentó el Esposo, retira el cadáver la Facultad de Medicina 


\section{Mirada hasta tornarse (In)visible: la historia cínica}

Inicialmente puede advertirse la multiplicidad de miradas y de voces que recaen sobre Ella: la policial que la considera "loca", la del médico que realiza la admisión, la del esposo que acompaña en el ingreso y responde al interrogatorio, quien solicita el corte de pelo, la tía que lo autoriza y quien ejecuta dicho corte. Una vez internada, puede leerse la mirada psi y la mirada clínica que examinan minuciosamente la mente y el cuerpo (respectivamente) de Ella.

Al indagar la evolución de la Historia Clínica de Ella visualizamos cómo gradualmente se registra un deterioro físico y psíquico, sin que medie tratamiento alguno o reflexión sobre dicha situación, cada nuevo registro no parece considerar la lectura de los previos. Aparece la descripción de comportamientos cada vez más "agresivos", "eróticos", "escapistas", que conducen al diagnóstico de "esquizofrenia", esto es, a la asignación de una categoría diagnóstica que fija una identidad a la paciente.

Cabe señalar que está ausente una lectura que componga y organice cada una de las partes en las que fue dividida Ella. Del mismo modo que está ausente una lectura que dé cuenta del tiempo, un malestar que avanza, unos síntomas que se repiten, un tratamiento ausente, una escucha y palabras anuladas y un cuerpo que se adelgaza hasta perder el último aliento que le queda para salir.

Ella fallece de un paro cardio-respiratorio no traumático, como casi todos lo haremos, como nadie en última instancia lo hace. La propia categoría diagnóstica condensa una nulidad y borramiento de una trayectoria de vida en el hospital densamente significativa que es eliminada por completo en el certificado de defunción que pasa a engrosar la historia clínica de un cadáver que, irónicamente será "útil" como deseaba Ella "ser útil" entre los estudiantes de medicina, condenada a ser observada eternamente, nombrada, descripta como un cuerpo sin voz que no tiene nombre (o no el suyo al menos) ni historia. Quizá lo más vivo que hallamos sean las palabras que gritan por salir, atrapadas en esta historia cínica.

Estos aspectos son una constante en gran parte de las historias clínicas de mujeres que ingresan hasta que su deterioro psico-fisico, y en este caso también la edad, las conduce a la muerte.

\section{Una lectura y análisis}

La historia clínica condensa diversas construcciones de la enfermedad y de la enferma, cada uno de los relatos transcriptos registran la descripción de Ella a partir del ojo clínico del médico, de la capacidad de penetración psíquica del psiquiatra, de la búsqueda por el mantenimiento de un orden el jurídico, tales relatos no parecen cruzarse, siquiera puede leerse conexiones. La forma en que es tratada Ella responde a las ideas que se establecen con la medicina moderna: una clara separación entre cuerpo/psiquis, biológico u orgánico/ espiritual o mental, lo cual permitirá establecer solo ciertas conexiones entre ambas y, una vez descartada una posible etiología orgánica, la constitución y el estado psíquico ocuparán toda la lectura y análisis de los signos y síntomas identificados.

En esta instancia es pertinente introducir los tres cuerpos como analizadores de la realidad, atravesados por la categoría de género. Así, a nivel del cuerpo individual puede leerse la relación de Ella con su esposo y su percepción subjetiva de sí misma al vincular su padecimiento con "La angustía que le produce el no hacer lo que ella quería (estudiar, ser útíl)". Que se traduce de inmediato a nivel del cuerpo 
social encontrar que los roles estereotipados de género impuestos sobre su cuerpo: el ser esposa-madre-cuidadora-ama de casa, no le alcanzan. El incumplimiento o apartamiento de ciertas características estereotipadas establecidas socialmente para su género como lo afectivo, lo débil, lo reproductivo, lo emocional, lo delicado, lo romántico, lo maternal-cuidador, lo doméstico la convierten en "loca". Estas características culturales atribuidas a los cuerpos sexuados como si fueran naturales o instintivas, producen prácticas, subjetividad y padecimientos, a la vez que ciertos procedimientos, acuerdos y prácticas que se cuelan en las teorías, registros, prácticas y modos de pensar médicos: "no se ocupa de sus quehaceres domésticos", "Desafectivizada hasta con sus híjos", "Erótica",

Entre otras características que son registradas como signos y síntomas de enfermedad. En este registro se nota el carácter moral de la definición de síntomas psiquiátricos que naturaliza los estereotipos de mujer=madre=ama de casa=cuidadora=amorosa e interpreta como síntoma patológico el apartamiento de esta norma. Esto no implica que la persona posiblemente pudiera estar sufriendo o requiriendo algún cuidado específico, sino que en aquel sentido la psiquiatría ha venido a legitimar el encierro de todas aquellas que se aparten de la norma, de la razón, de lo productivo, de lo esperable para su género; un cuerpo político al que hay que cuidar y mantener en orden.

El análisis de las biografías y obras de mujeres escritoras (las llamadas "locas ilustres"), dio lugar a una corriente que ha interpretado la locura como un refugio, un lugar donde protegerse de las estrategias opresoras del sistema patriarcal, una vía de escape de la domesticidad, un instrumento de resistencia, que permitiría actuar fuera de los estereotipos y roles establecidos. Ante estas interpretaciones, cabría señalar que las mujeres que rechazaron la domesticidad o el dominio económico y sexual de los varones, así como las activistas políticas, optaron por transgredir los roles de género que se le habían asignado; no "optaron" por la locura. Es decir, debajo de la locura pueden encontrarse signos de resistencia y rebelión, no tanto en relación a que la locura en sí fuese un instrumento para ello, sino a que la locura se convirtió en una "capa» que pretendía ocultar, bajo las etiquetas de las distintas patologías, conductas y pensamientos que transgredían el orden establecido, asociándose, no pocas veces, con la peligrosidad sexual. Por tanto, podría ser considerada una etiqueta impuesta por agentes profesionalizados de un sistema de dominación, los psiquiatras (Ruiz Somavilla \& Jiménez Lucena, 2003: 10). El motivo de encierro alegado para Ella en la orden judicial, que remite al "gremíalismo" y "síndicalismo" o, en la historia clínica, "el no hacer lo que ella quería (estudiar..." son algunas pistas para interpretar el encierro ante la resistencia a la vida doméstica, aunque es clave la expresión final "Ya no tengo alucinaciones ni ilusiones"; esta expresión alude a la claridad de haber hallado que para salir, cualquier vinculación con el orden de la fantasía o el deseo por ser algo o alguien diferente debieran eliminarse. Y, cuando desde lo discursivo Ella está dispuesta a apagar el deseo, la fantasía o la ilusión, también se apaga el cuerpo, aun pareciendo evidente la conexión entre ambos eventos, Ella muere de un "paro cardio-respiratorio", el deseo que se apaga no es más que el registro de una voz que no se escucha.

Como señala Taussig (1992) la enfermedad adquiere una "objetividad fantasmal" al negar las relaciones humanas encarnadas en los síntomas, signos y terapias, se mistifica y reproduce una ideología y política a modo de ciencia de "cosas reales» ocurriendo una cosificación biológica y física donde las cosas toman vida propia, separadas del nexo social que las originó, borrando el significado social y moral los signos y síntomas de la enfermedad.

\section{A modo de cierre}

La denominada "locura" tiende a ocupar el lugar de lo enigmático, lo distinto, lo otro que, por lo tanto, las sociedades tratan de significar de acuerdo a sus valores, ideologías y significaciones predominantes (Sy, 2015). La evolución de los tratamientos para mujeres con 
sufrimiento psíquico ha ido de la mano de las características socioculturales y políticas de la sociedad en que estos se desarrollaban, patologizando aquellas actitudes que se apartan de las esperadas para los roles de género establecidos. Así, los diagnósticos y tratamientos se constituyen en un reflejo de la sociedad implicada, de los supuestos epistémicos y paradigmas vigentes.

En nuestro análisis observamos cómo, en un periodo de casi cincuenta años, aparecen signos y síntomas que representan claramente el registro del comportamiento de mujeres que se apartan del rol de género esperado para la época. Si bien podemos señalar que durante tal periodo de tiempo los tratamientos administrados y, aún más, las teorías y corrientes de la psiquiatría se transforman, observamos que en la práctica clínica -que se traduce en el registro de signos y síntomas- persiste la adjetivación de la conducta femenina reprobable para la época, como si se tratara de un hecho observable "científicamente", antes que una apreciación construida socio-culturalmente en un momento histórico particular.

Cabría preguntarse a partir de este análisis, en qué medida estos estereotipos de género condicionan hoy en día los diagnósticos que realiza la psiquiatría como una "realidad" acrítica que habilita el diagnóstico e internación de las mujeres como "insanas".

\section{Referencias}

Bullard, A. (2001). The truth in madness. Colonial doctors and insane women in French North Africa, South Atlantic Review, 66 (2), pp. 114-132.

Burin M. y Meler I. (2010). Género y Familia: poder, amor y sexualidad en la construcción de la subjetividad. Editorial Paidos. Buenos Aires, Argentina.

Chesler, P. (1972). Women and Madness, New York: Avon Books.

Davies, K. (1996). "Sexing the mind"? Women, Gender and Madness in nineteenth-century welsh asylums, Llafur, 7 (1), pp. 29-40.

Kromm, J.E. (1994). The Feminization of Madness in Visual Representation, Feminist Studies, 20 (3), pp. $507-535$. Lock M, Scheper N. (1990). A critical-interpretative approach in medical anthropology: rituals and routines of discipline and dissent. In Johnson TM, Sargent CF (editors) Medical anthropology: Contemporary theory and method. New York: Praeger. Pp. 47-72.

Ruiz, M.J. y Jiménez, I. (2003). Género, mujeres y psiquiatría: una aproximación crítica. En: Frenia, Vol. 3(1) pp. 7-29. Scott, J. (1993). El género: una categoría útil para el análisis histórico. En: Marta Lamas (comp). El género, la construcción cultural de la diferencia sexual. México. PUEG: UNAM.

Showalter, E. (1985). The Female Malady: Women, Madness, and English Culture, 1830-1980, New York: Pantheon. Smith, C. (1972). The Histerical Woman: Sex, Roles and Role Conflict in 19th Century America, Social Research, 39 (4) pp. 652-678.

SY A. (2015). De la Literatura a la Historia: Cuando la locura se convierte en desviación social. Estudios filológicos, (55), pp. 129-141. Disponible en: http://www.scielo.cl/scielo.php?script=sci_arttext\&pid=S007117132015000100008\&Ing=es\&tIng=es. 10.4067/S0071-17132015000100008

Taussig M. (1922). La reificación de la conciencia del paciente. En: Un gigante en convulsiones. El mundo humano como sistema nervioso en emergencia permanente. Barcelona: Gedisa; 1992, pp. 111-143. 